\title{
Haemosporidian infections in captive exotic glossy starling Lamprotornis chalybaeus in Hong Kong
}

\author{
Ilan Paperna ${ }^{1}$ and Paolo Martelli ${ }^{2}$ \\ ${ }^{1}$ Department of Animal Sciences, Faculty of Agriculture, Food and Environmental Quality, Hebrew University of Jerusalem, \\ Rehovot 76-100, Israel; \\ ${ }^{2}$ Veterinary Department, Ocean Park, Aberdeen, Hong Kong
}

Key words: Haemosporidia, Plasmodium octamerium, Plasmodium relictum, Haemoproteus pastoris, blood parasites, Lamprotornis chalybaeus, Sturnidae

\begin{abstract}
A greater blue-eared glossy starling Lamprotornis chalybaeus Ehrenburg from a large flight aviary in Hong Kong was found on post mortem to be infected with Plasmodium octamerium Manwell, 1968, Plasmodium cf. relictum (Grassi et Feletti, 1891) and Haemoproteus cf. pastoris Mello, 1935. Descriptions of their morphology are provided as none of the examined parasites fully concord with their type (or neotype) material descriptions. Plasmodium octamerium has been recorded in avian hosts from geographically distant locations, suggesting that infection in imported hosts may persist in a chronic state for a long period. This Plasmodium species as well as P. relictum are evidently not fastidious in choice of passeriform hosts and are transmitted by ubiquitous domestic mosquito vectors, apparently facilitating their proliferation among zoo and aviary inhabitants. The Haemoproteus infection appears to be conspecific with $H$. cf. pastoris reported from a myna (Acridotheres tristis) in Singapore. Mynas are also common in Hong Kong, which suggests a possible cross-transmission of infection between these two starlings.
\end{abstract}

A greater blue-eared glossy starling Lamprotornis chalybaeus Ehrenburg of African origin, obtained from an aviary in Germany and kept for 10 years in an aviary in Hong Kong, died on 16 October 2005. Three haemosporidian parasites were identified from blood smears prepared ante and post mortem: Plasmodium octamerium Manwell, 1968, Plasmodium cf. relictum (Grassi et Feletti, 1891) and Haemoproteus cf. pastoris Mello, 1935.

Manwell and Rossi (1975) in their communication on blood protozoa of imported birds to the USA list $P$. octamerium as well as $P$. circumflexum and $P$. nucleophilum from L. chalybaeus. Bennett and Herman (1976) report infections with Haemoproteus sturni (syn. of $H$. pastoris - see Valkiunas 1997), P. relictum and a species of Leucocytozoon in L. chalybaeus from Kenya. Ashford et al. (1976) report infections with Haemoproteus sp., Plasmodium relictum, and Leucocytozoon sp. in Ethiopia.

In the present communication, morphology of the reported parasites is described and possible routes of transmission among exotic captive birds are discussed.

\section{MATERIALS AND METHODS}

The starling's blood was examined prior to (withdrawn from the brachial vein) and after its death (withdrawn from the heart). Blood films were fixed in absolute methanol and stained with Giemsa (15\% in phosphate buffer, $\mathrm{pH} 7.2)$ for 1 hour. Microscopic examination included a check of at least 50,000 erythrocytes at $\times 1,000$ (oil immersion) magnification. For $H$. cf. pastoris was calculated the Nuclear Displacement
Ratio (Bennett and Campbell 1972) - 2X / X+Y (X = distance between the erythrocyte wall and its nucleus; $\mathrm{Y}=$ distance between the erythrocyte wall and its nucleus on the infected side).

\section{RESULTS}

\section{Description of the parasites}

Plasmodium (Giovannolaia) octamerium Manwell, $1968 \quad$ Figs. 1-14

Parasites were seen usually in mature erythrocytes, exceptionally (trophozoites) in reticulocytes. They neither deformed the erythrocyte, nor displaced its nucleus. The level of parasitaemia was $4 \%$ (calculated from 6,080 erythrocytes); $46 \%$ were trophozoites. Trophozoites appeared alongside the erythrocyte nucleus, but did not touch it. They already contained several pigment granules (Figs. 1-4). In young meronts, half of their volume was occupied by the single nucleus, and their cytoplasm contained many translucent vacuoles (Fig. 5). The only meront with two nuclei traced, was longer than the erythrocyte nucleus and was extending alongside it. This meront contained an aggregate of pigment and three vacuoles staining conspicuously blue (Fig. 6). Only few meronts with eight or less nuclei were traced (Figs. 7, 8). Differentiated meronts (segmenters) contained 10-16 nuclei (i.e., a progeny of 10-16 merozoites), arranged into a string of paired nuclei, extending alongside and exceeding in length the host nucleus (Figs. 9, 10); few rounded up into a polar position (Fig. 11). The chain of nuclei was usually interrupted by a 

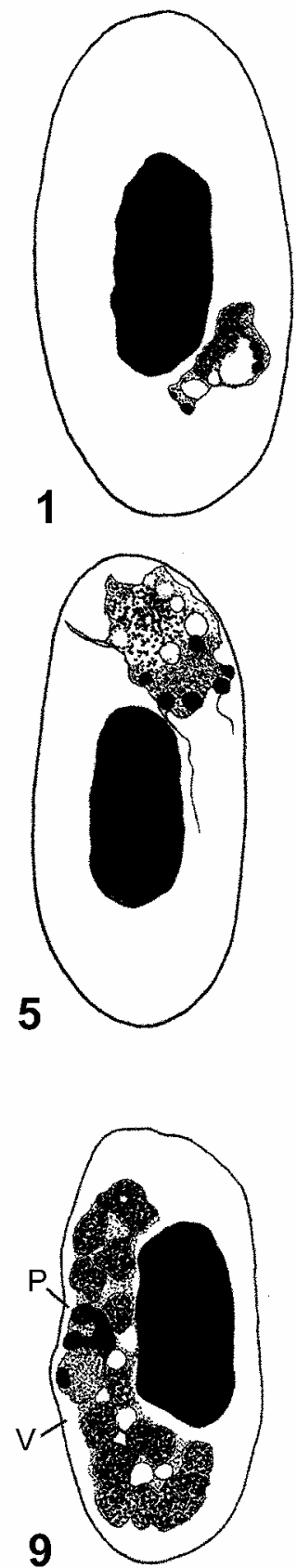
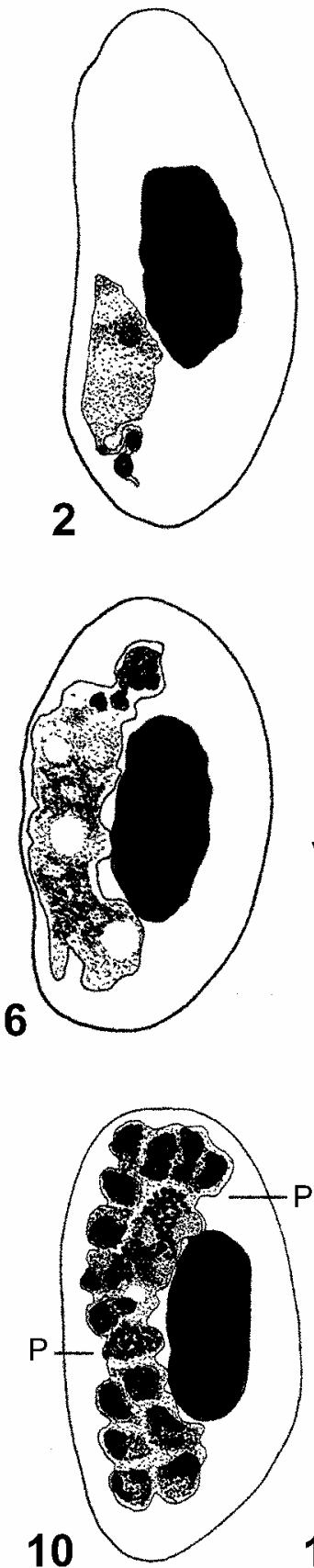
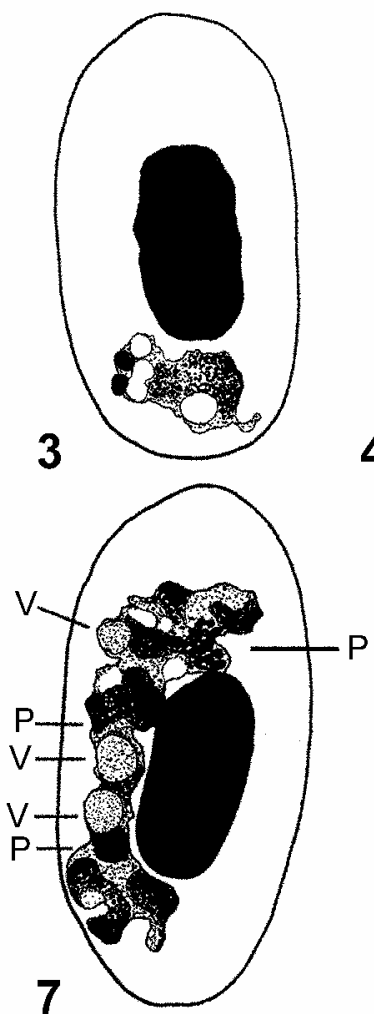

7

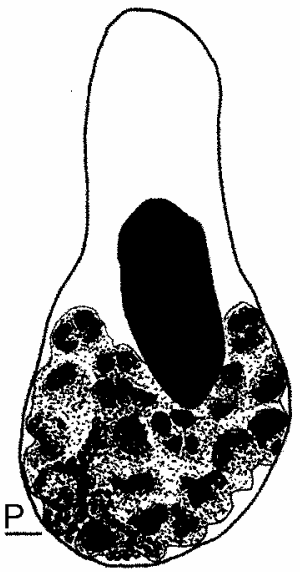

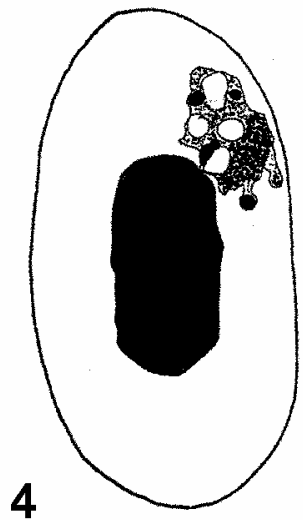

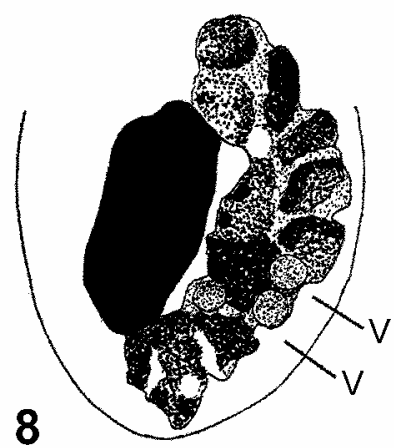

$2 \mu \mathrm{m}$

Figs. 1-11. Plasmodium octamerium from Lamprotornis chalybaeus. Figs. 1-4. Trophozoites. Fig. 5. Young uninucleate meront. Fig. 6. Meront with two nuclei. Figs. 7, 8. Meronts with up to eight nuclei. Figs. 9, 10. Mature schizonts with 14-16 nuclei. Fig. 11. Meront with 16 nuclei in polar position. $\mathrm{P}$ - pigment; $\mathrm{V}$ - vacuole.

cytoplasmic segment where pigment aggregated, often accompanied by one or more vacuoles (globules), staining conspicuously faint blue (Figs. 7-9).

The gametocytes were elongate, exceeding the erythrocyte nucleus in length. The mid-part of both the macro- (Figs. 12, 13) and microgametocytes (Fig. 14) was inflated and either displaced the erythrocyte nucleus or descended to a polar position. The pigment was either aggregated or scattered.

\section{Plasmodium (Haemamoeba) cf. relictum (Grassi et} Feletti, 1891)

Figs. 15-23

Infection was sparse; parasitaemia was $0.5 \%$ (trophozoites, if present, could not be distinguished from $P$. octamerium). Observed meronts contained 5 to 8 nuclei leaving in between wide patches of cytoplasm (Figs. 15-19). Pigment either aggregated in patches or in scattered grains. The meronts and particularly the gameto- 

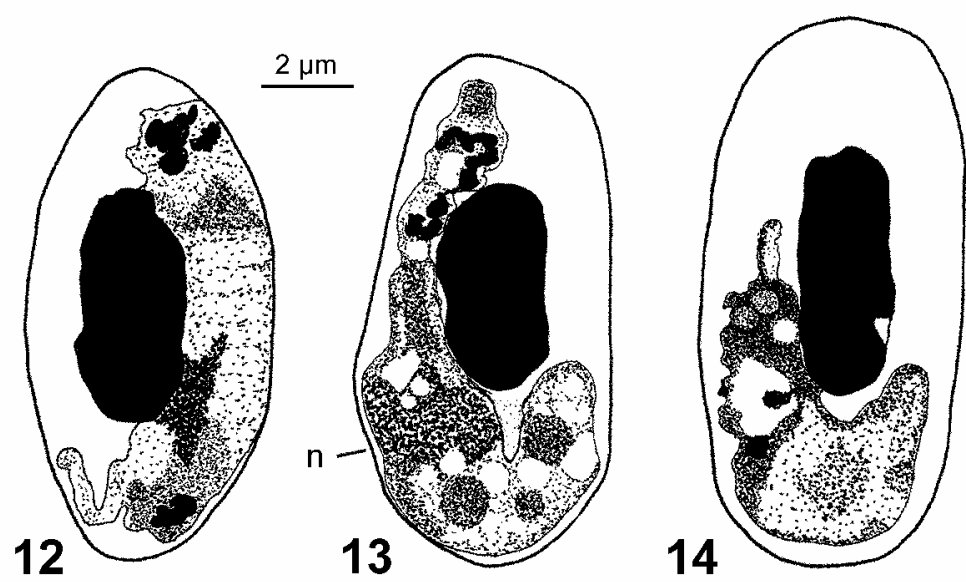

Figs. 12-14. Plasmodium octamerium from Lamprotornis chalybaeus. Figs. 12, 13. Macrogametocytes. Fig. 14. Microgametocyte in polar position. $\mathrm{n}$ - nucleus.

cytes occupied up to about three quarters of the erythrocyte space, while displacing the nucleus to apical or lateral position; in the process the erythrocyte rounded up (Figs. 20-23).

One erythrocyte contained a nine-nucleate $P$. cf. relictum meront and a microgametocyte of either $P$. cf. relictum (more likely) or $P$. octamerium; the erythrocyte nucleus was strongly displaced to lateral position.

\section{Haemoproteus cf. pastoris Mello, 1935 Figs. 24-31}

Infection was low and detected only in the set of blood films drawn ante mortem. The blood films contained only macrogametocytes in several stages of differentiation. The erythrocyte became enlarged from an early stage of infection (Figs. 25, 26). Young ellipsoid macrogametocytes were 6.0-6.4 $\times 2.4 \mu \mathrm{m}$ (Figs. 25, 26 ), those slender, elongate were 8.7-9.6 $\times 1.8 \times 2.2 \mu \mathrm{m}$ (Figs. 27-29) and those large, mature were 10.7-11.8 $\times$ 2.1-3.3 $\mu \mathrm{m}$ in size (Figs. 30, 31). With maturation, the infected erythrocyte became increasingly deformed and its nucleus became displaced. The nuclear displacement increased from a ratio of 1 in young ellipsoid-stage infection to $0.53-0.37$ in infections of slender stages to $0.33-0.30$ in erythrocytes with differentiated (or mature) macrogametocytes.

\section{DISCUSSION}

Plasmodium octamerium. It was first described in a bird of African origin, Vidua macroura (Pallas), of the family Estrilidae, obtained from a pet shop in the USA (Manwell 1968). Manwell's (1968) experimental infection of canaries yielded about $80 \%$ segmenters with 8 nuclei. In a different experimental host, Spizella arborea, infection consisted of many segmenters with 10 , over 12 to over 16 merozoites. Valkiunas (1997) in his account on $P$. octamerium from Manwell's neohapantotype (Nos. 645 and 646) from "Sturnus sp." (= L. chaly- baeus?) reports meronts with 6-24 merozoites. Neither Manwell (1968) nor Valkiunas (1997) mention the presence of faint-blue-staining vacuoles. Such vacuoles could, however, become less distinct in long stored specimens. Among species of the subgenus Novyella the blue-green-staining body was described as a globule due to its refractory properties (Mohammed 1958); in our $P$. octamerium the vacuoles or globules were not conspicuously refractile. Valkiunas (1997) also reports infections in immature erythrocytes, not mentioned earlier. The flagellum-like appendices, although not mentioned in earlier descriptions are not exceptional among species of the subgenera Giovannolaia and Novyella (Valkunias 1997). In spite of several discrepancies between the available descriptions and the presently described parasite, it is most likely P. octamerium.

Plasmodium cf. relictum. A recent molecular study confirmed that $P$. relictum is not fastidious in choice of passeriform hosts (Martinsen et al. 2006). Molecular data, however, also yielded ambiguous results: at least two distinct lineages were attributed to $P$. relictum isolates: GRW4 (Valkiunas et al. 2007) and SGS1 (Gill H. and Paperna I., unpubl.; Valkiunas G.A., pers. comm.). More molecular studies will be required to identify the boundaries between species included in the subgenus Haemamoeba Garnham, 1966 and the extent of conspecificity between infection with agents of morphology similar to that of Plasmodium relictum (see Landau et al. 2003) from diverse hosts.

Haemoproteus cf. pastoris. The gametocytes from the glossy starling blood were identical with $H$. cf. pastoris reported from a myna (Acridotheres tristis) in Singapore (Paperna et al. 2005, 2007). The gametocytes from both hosts differ from H. pastoris from Sturnus roseus, the type host (and other starlings, see Valkiunas 1997), mainly in having a heavy load of coarse pigment granules and in extremely displacing the erythrocyte nucleus already from premature stage of development. 

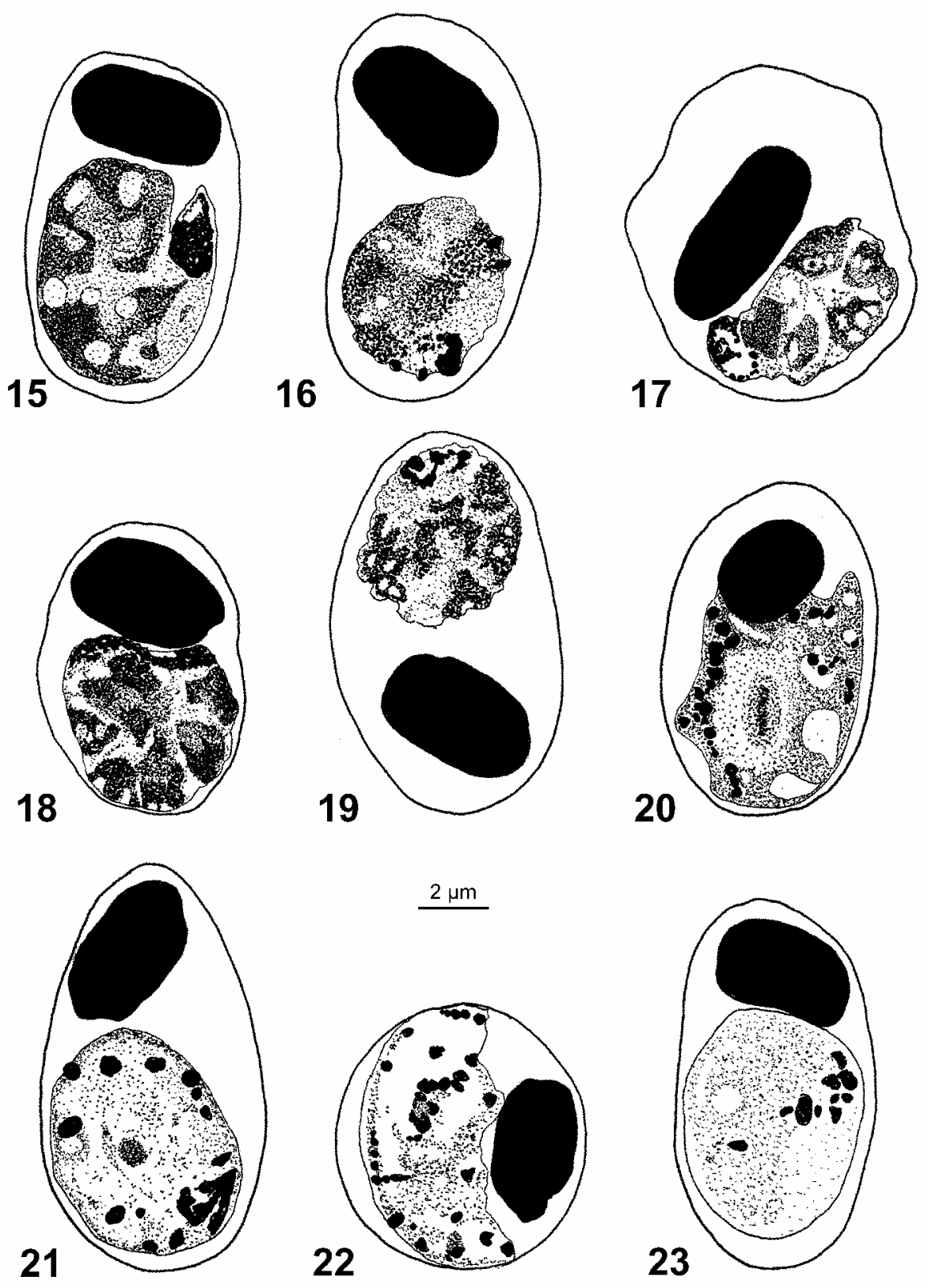

Figs. 15-23. Plasmodium cf. relictum from Lamprotornis chalybaeus. Figs. 15-19. Meronts. Figs. 20, 21. Macrogametocytes. Figs. 22, 23. Microgametocytes.

Infection in captive birds: caged pets, aviaries and Zoos. Plasmodium octamerium has been reported both from native L. chalybaeus (Bennet and Herman 1976) and from imported pet birds - Vidua macroura, its type host (Manwell 1968), and L. chalybaeus (Manwell and Rossi 1975, present communication). Its appearance in geographically widely separated avian hosts suggests that infection in the imported hosts may persist in a chronic state for a long period (perhaps years). Nonethe- less, the possibility that the infection was imported with its host from Germany 10 years ago is very remote. There are no data suggesting what species of culicine mosquitoes are capable of transmitting $P$. octamerium. Many species of avian Plasmodium are, however, nonfastidious in their choice of vectors and are capable to be transmitted by ubiquitous domestic mosquitoes (Culex pipiens) and in Hong Kong likely by Culex quinquefasciatus (Hong Kong Government 2005). 

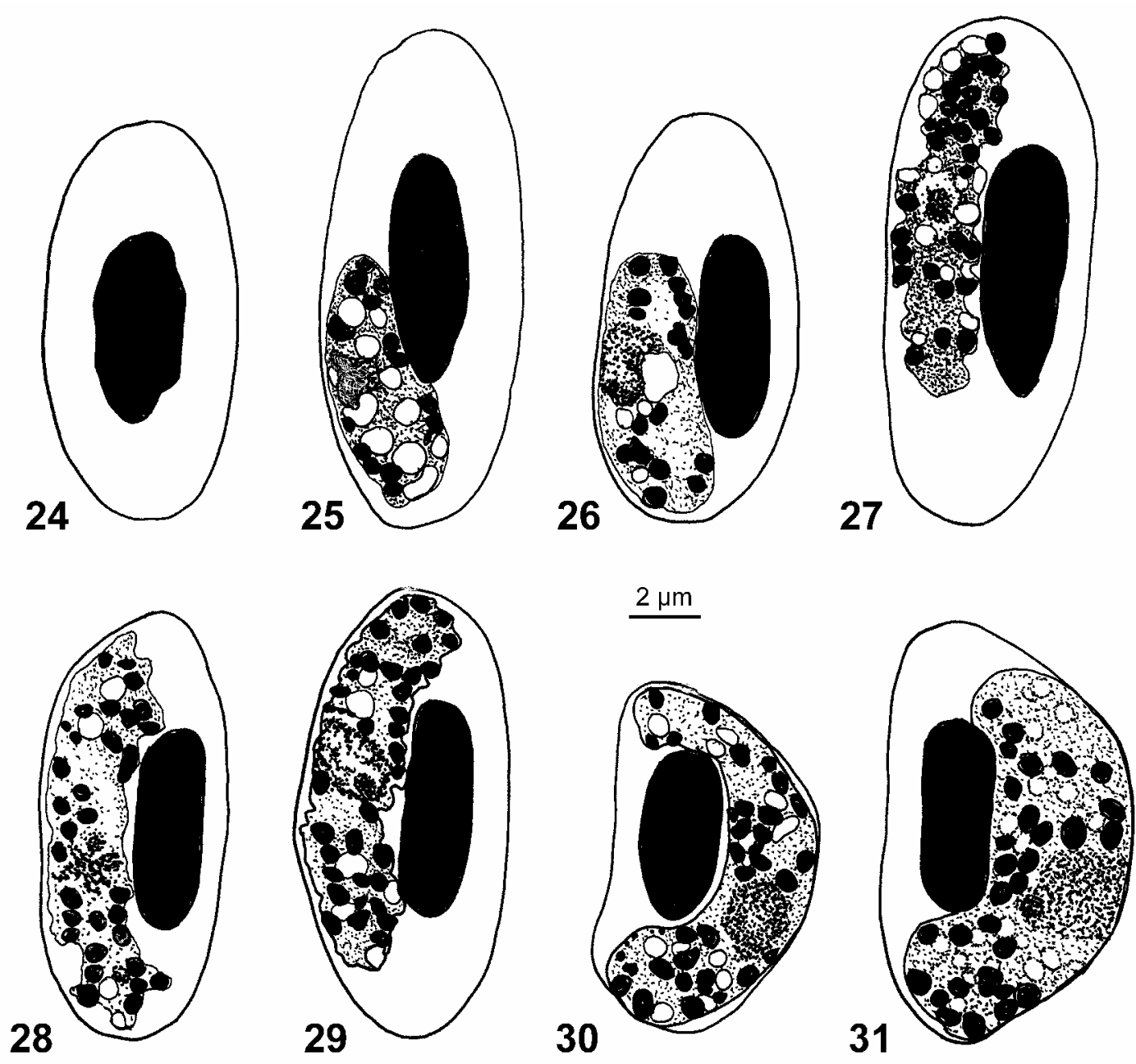

Figs. 24-31. Haemoproteus cf. pastoris from Lamprotornis chalybaeus. Fig. 24. Non-infected erythrocyte. Figs. 25, 26. Young macrogametocytes. Figs. 27-29. On-growing slender and elongate macrogametocytes. Figs. 30, 31. Mature macrogametocytes grossly distorting the host erythrocyte.

In Manwell and Rossi's (1975) list, P. relictum was considered rare, reported in only one bird. Fatal incidences due to $P$. relictum (or $P$. relictum-like species) hyperparasitaemia have been observed among captive birds (Beier et al. 1981). Infections with P. relictum were linked to mortalities among Zoo penguins (Stoskopf and Beier 1979, Graczyk et al. 1994). Available data, including molecular studies (McConkey et al. 1996), leave us uncertain as to whether the penguin parasites are conspecific with $P$. relictum found among passerine birds (Valkiunas 1997, Landau et al. 2003). The apparent low species specificity of $P$. relictum to avian hosts together with its capability of being trans- mitted by ubiquitous domestic vectors (such as $C$. pipiens) (see Valkiunas 1997) increases the risk of its proliferation among Zoo and aviary inhabitants.

The apparent conspecificity between the Haemoproteus infection agent from Singapore myna and that from the Hong Kong glossy starling L. chalybaeus suggests a cross-transmission between these two starlings. Myna is common in Hong Kong; it is an invasive bird which expands it distribution range throughout the warm zones of Asia, Africa and the Pacific.

Acknowledgement. We wish to thank Ms. Mickey Cheung for her technical help. 


\section{REFERENCES}

ASHFORD R.W., PALMER T.T., ASH J.S., BRAY R.S. 1976: Blood parasites of Ethiopian birds I. General Survey. J. Wildl. Dis. 12: 409-426.

Beier J., StrandBerg J., Stoskopf M.K., CRAFT C. 1981: Mortality in robins (Turdus migratorius) due to avian malaria. J. Wildl. Dis. 17: 247-250.

BenNETT G.F., CAMPBELL A.G. 1972: Avian Haemoproteidae. 1 Description of Haemoproteus fallisi n. sp. and a review of haemoproteids of the family Turdidae. Can. J. Zool. 50: $1269-1275$.

BENNETT G.F., HeRMAN C.M. 1976: Blood parasites of some birds from Kenya, Tanzania and Zaire. J. Wildl. Dis. 12: 5965.

GARNHAM P.C.C. 1966: Malaria Parasites and Other Haemosporidia. Blackwell Scientific Publications, Oxford, 1114 pp.

GRACZYK T.K., CRANFIELD M.R., MCCUTCHAN T.F., BICKNESE E.J. 1994: Characteristics of naturally acquired avian malaria infections in naïve juvenile African blackfooted penguins (Spheniscus demersus). Parasitol. Res. 80: 634-637.

HoNG Kong GOVERnMENT, Food and Environmental Hygiene Department 2005: Mosquitoes of Hong Kong, August 2005.

LANDAU I., Chabaud S., BerTani S., SNOUNOU G. 2003: Taxonomic status and re-description of Plasmodium relictum (Grassi \& Feletti, 1891), Plasmodium maior Raffaele, 1931, and description of P. bigueti n. sp. in sparrows. Parassitologia 45: 119-123.

MANWELL R.D. 1968: Plasmodium octamerium n. sp., an avian malaria parasite from the pintail whydah bird Vidua macroura. J. Protozool. 15: 680-685.

MANWELL R.D., Rossi G.S. 1975: Blood protozoa of imported birds. J. Protozool. 22: 124-127.
MARTINSEN E.S., PAPERNA I., SCHALL J.J. 2006: Morphological versus molecular identification of avian Haemosporidia: an exploration of three species concepts. Parasitology 133: 279288.

MCCONKEy G.A., LI J., Rogers M.J., SEeley D.C. JR., GRACZYK T.K., CRANFIELD M.R., MCCUTCHAN T.F. 1996: Parasite diversity in an endemic region for avian malaria and identification of a parasite causing penguin mortality. J. Eukaryot. Microbiol. 43: 393-399.

MoHAmmeD A.H.H. 1958: Systematic and Experimental Studies on Protozoal Blood Parasites of Egyptian Birds. Vol. 1. Cairo University Press, Cairo, Egypt, 165 pp.

PAPERNA I., SOH M.C.-K., YAP C.A.-M.: Haemosporozoan parasites found in birds in peninsular Malaysia, Singapore, Sarawak and Java. Raffles Bull. Zool. (In press.)

PAPERNA I., SOH M.C.-K., YAP C.A.-M., SOHDI N.S., LIM S.L.H., Prawiradilaga D.M., Nagata H. 2005: Blood parasite prevalence and abundance in the bird communities of several forested locations in Southeast Asia. Ornithol. Sci. 4: 129138.

StOSKOPF M.K., BeIER M.K. 1979: Avian malaria in African blackfooted penguins. J. Am. Vet. Med. Assoc. 175: 944-947.

VALKIUNAS G.A. 1997: Bird Haemosporidia. Acta Zool. Lithuan. 3-5: 1-608. (In Russian with English summary; English translation 2005: Avian Malaria Parasites and other Haemosporidia. CRC Press, Boca Raton, 946 pp.)

VALKIUNAS G.A., ZEHTINDJIEV V P., HeLLEGREN O., LLIEVA M., ISEZHOVA T.A., BENSCH S. 2007: Linkage between mitochondrial cytochrome $\mathrm{b}$ lineage and morphospecies of two avian parasites with a description of Plasmodium ashfordi. Parasitol. Res. 100: 1311-1322.

Accepted 25 July 2007 